\title{
A Survey on Search Engine Optimization (SEO)
}

\author{
Anis ISMAIL and Firas ABDALLAH
}

\begin{abstract}
With the growth of Internet in the recent years, search engine such as Google, Bing and Yahoo are becoming more and more important. The role of the search engines is to index billions of web pages and display only the most relevant results for a given search query. When creating the website, many webmasters forget to take into consideration an essential factor, which is making the world aware of their website. Most of times, main focus is set on making the website as user-friendly as possible, stable, fast and secure. In this paper, we present may techniques that are sometimes useless. The aim of these techniques is to verify if the website does not have visitors or simply not found.
\end{abstract}

Keywords - Internet, Google, Bing, Yahoo, search engines, SEO.

\section{INTRODUCTION}

Search engine is an application used to help find information on the internet. The search engine is usually accessed through a page on a website that allows a user to search the content of billions of websites on the web by entering a set of keywords into a search textbox [1]. The keywords submitted by the user will then be matched against the cached or indexed pages in the search engine's database and a list of references that match the keywords or the keywords' criteria will be returned [2]. Search engines index new web pages and update existing ones regularly to provide a quicker and more efficient search.

Search engines are not only used to search for text content. They can be used to search for images, videos, applications, books and much more. In addition, some search engines such as Google, which is the leading search engine today, allows the search to be filtered by time, country and city.

Search Engine Optimization (SEO) $[1,4,5]$ is the process of improving the structure of a website and promoting it in order to make it visible in search engines, improve its rank and increase the number of visitors. There are many different ways to apply SEO for a website, it ranges from the keywords on the web page to the way other websites are linking to the site. The below figure shows some additional ways of applying SEO to a website, for example someone can target different set of keywords on their webpage, others can promote their website on social medias such as Facebook or twitter, or they can use analytics such as Google analytics to track the behavior of users, understand their needs and modify the website according to those needs.

Anis ISMAIL. Université Libanaise, Institut Universitaire de Technologie, Saida-LIBAN , (e-mail anismaiil@yahoo.com)

Firas ABDALLAH . Université Libanaise, Institut Universitaire de Technologie, Saida - LIBAN , (e-mail Firas.Abdallah@ul.edu.lb)

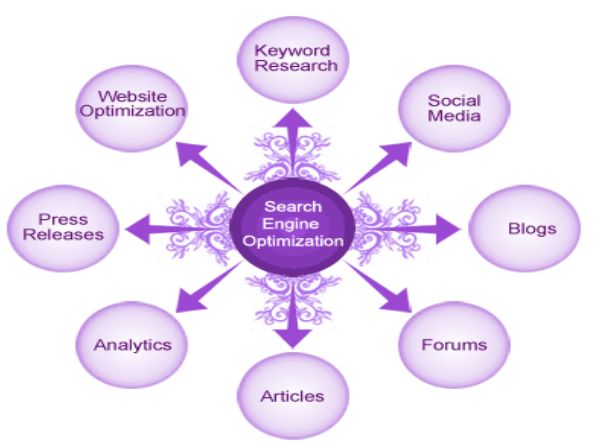

Fig. 1.Different SEO Techniques [3]

Most of the times, SEO is just simply a method of building the structure of the website in a way that it will allow search engines to understand what the website is all about. The majority of the web visitors are driven to websites by search engines such as Google, Bing and Yahoo. Despite the fact that social media such as Facebook, Twitter and even Youtube and many others can still be the source of visitors to a website, search engines remains the primary method of locating and visiting a website for most of the internet surfers. This applies to all types of websites, such as ecommerce, blogs, forums, social media and news websites.

Not only most of the web visitors are driven by search engines, but search engines are amazing in a way that they send targeted traffic to a website, in other words it sends users that are looking for what the website is offering. This is why SEO is important, it helps a website to be found by search engines, which in turn send targeted traffic and generates higher revenues in case of ecommerce websites.

According to a study made in 2012, there are around 54,000 search queries per second done on Google alone. Search queries which are the words that a user enters into the search box of a search engine are very valuable. That's because those keywords used in the search query are the ones generating the targeted visitors. That's why choosing the right keywords for a webpage or a website play a very important role in SEO.

In addition to making search engines understand the content of web pages, SEO also helps in increasing the ranking of a website in order to make it more visible in search engines and to help the users find it easily. The Internet is growing very fast and is becoming increasingly competitive, and the webmasters that applies SEO techniques (figure 1) will have an advantage and will be rewarded by more visitors and web traffic. The world of SEO is very sophisticated, but most webmasters can easily understand the fundamentals. Even the slightest amount of knowledge can make a difference. 


\section{EXISTING SOLUTIONS}

Different types of solution exist and are developed, but few are practical enough to enhance the search engine optimization of a website or provides helpful tips on how to improve the website. In this paragraph, we will introduce these existing solutions. Several solutions exist for the moment on the market. They are "Webmaster tools", "Google Analytics", "Google PageSpeed Tool", "Xenu Link Sleuth", "Google AdWords Keyword Tool", "Raven Tools" and "Alexa".

\section{A. Google and Bing Webmaster tools}

Webmaster tools such as [6, 7] Google Webmaster Tools and Bing Webmaster Tools are a set of free tools that give an important insight about a website. The tools allow a webmaster to see what search queries (figure 2) led to the site being listed in the search engine results pages, the number of impressions (how many users have seen the website in the results), the number of clicks, the click through rates and the average position in the results.

\begin{tabular}{|lcccc|}
\hline Query & Impressions A & Clicks & CTR & Avg. position \\
\hline $\begin{array}{l}\text { facebook } \\
\text { registration plugin }\end{array}$ & 500 & $<10$ & - & 8.0 \\
$\begin{array}{l}\text { most used linux } \\
\text { commands }\end{array}$ & 200 & 22 & $11 \%$ & 5.4 \\
$\begin{array}{l}\text { facebook app } \\
\text { tutorial 2012 }\end{array}$ & 110 & 12 & $11 \%$ & 6.8 \\
\hline
\end{tabular}

Fig.2. Google Webmaster Tools - Search Queries [7]

In addition, the webmaster tools provide a list of all the internal links, eg. links within the website pointing to other pages in the same website, such as navigation button and menus. A list of broken links such as links to a non existing page. A list of websites linking to our website.

Furthermore, webmaster tools (figure 3)allows a webmaster to submit a sitemap which contains a list of all the pages of the website and their links in order for the search engine to be able to index all the web pages. Sitemaps also contains the date that the page was last modified, the priority of the page and how often the page is updated, this tells the search engines how often they should index that specific page and update their database.

This message is not indicative of any problem in your site. It is simply to inform you that the number of clicks that one of your pages receives has increased recently. If you have just added new content, this may indicate that it has become more popular on Google. The number of clicks that your site receives from Google can change from day to day for a variety of factors, including automatic algorithm updates.

Fig. 3. Google Webmaster Tools - Big Traffic Change Notification [7]

Also, webmaster tools offers many other tools such as statistics on how the search engine is indexing the site, notifications containing crawling errors or ranking improvement, setting crawling rate and time and checking the website for malware.

\section{1) Advantages}

The first product we review are the Webmaster tools. Google and Bing Webmaster tools are one of the most important SEO tools available. They provide a lot of insights such as the search queries (keywords) that are being searched by the users and are leading to the website, the daily, weekly and monthly impressions, the clicks from the search engine to the website, the internal links within the website and the outbound links (the links from other websites linking to our site).

In addition, webmaster tools [7] allow a webmaster to submit a sitemap containing all the links of the website and how often those links should be indexed or re-indexed, this is a great way to guarantee that all the pages of the website are being indexed, which can be very useful for dynamic websites such as ecommerce websites where many new pages are updated and added every day.

Also, webmaster tools provide many helpful features such as sending notifications when errors occur that prevents the search engine from crawling a specific page, and the ability to manually submit a URL to index or re-index it. On top of all that, webmaster tools are totally free to use.

\section{2) Disadvantages}

The disadvantages of Google and Bing Webmaster Tools are the following. It does not work with all search engines, for example, Google Webmaster Tools only provide insights about the search queries or keywords used on Google. Usually search engines with a low market share do not have their own Webmaster Tools, so optimizing those websites with the help of Google or Bing Webmaster Tools will not work, and even though those search engines have a low market share, they can still be a big source of traffic to the website.

Although Google and Bing Webmaster Tools are free to use, but they require a verification of the domain name before they can start gathering and displaying information and insights. There are many ways to verify the domain name but the process of verification might be hard and challenging for someone without a technical background.

Google and Bing Webmaster Tools does not collect and display information immediately, it can take up for a week for some data to start showing up which might slow down the process of analyzing and improving the website.

\section{B. Google Analytics}

Google Analytics $[8,9]$ is a service offered by Google. It provides webmasters and marketers details about the visitors of the website, such as the number of visitors during a time frame, the visitor's location, time of the visit, average visit duration, new visitor or returning visitor and bounce rates (figure 4).

\begin{tabular}{|c|c|c|c|c|c|}
\hline Source & Visits & Pages /Visit & $\begin{array}{l}\text { Avg. Visit } \\
\text { Duration }\end{array}$ & \% llew Visits & Bounce Rate \\
\hline 1. facebookcom & 4,015 & 3.43 & 00.04 .40 & $19.18 \%$ & $40.00 \%$ \\
\hline 2. apps.tacebook.com & 3,363 & 3.53 & 00:03:58 & $4523 \%$ & $39.01 \%$ \\
\hline 3. igrab.me & 927 & 3.37 & $00: 04: 39$ & $8.41 \%$ & $46.28 \%$ \\
\hline 4. google.com.lb & 758 & 3.43 & 00:04:08 & $871 \%$ & $39.18 \%$ \\
\hline 5. m.lacebook.com & 299 & 2.50 & $00: 02: 49$ & $21.40 \%$ & $51.84 \%$ \\
\hline
\end{tabular}

Fig. 4. Google Analytics - Referring Websites [10]

In addition, it can provides information about the referring 
sites that is from which website the user has come from. The referring sites can be any website, such as Google, Facebook, Twitter, blogs and forums. The below picture displays a list of referring sites and the number of visitors from those websites as it appears in Google Analytics.

Another important feature in Google Analytics is the ability to set a goal, measure conversion and sales. By using goals, a webmaster can track how many visitors have reached that goal or converted, and adjust the website accordingly in case the number was very low. Ecommerce tracking (figure 5) provides details about every transaction, and the total revenue.

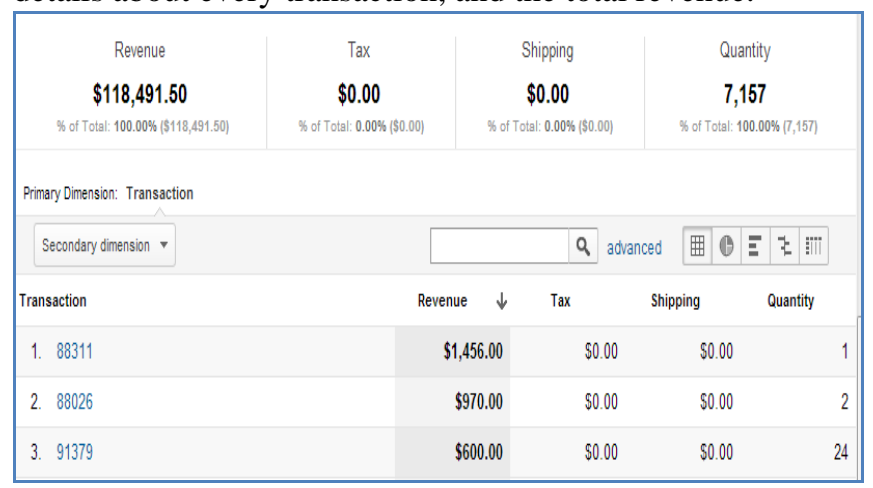

Fig. 5. Google Analytics - Ecommerce Tracking [10]

Furthermore, Google Analytics [9] provides detailed information about the browser being used and its version, the operating system of the user such as Windows, iOS, Linux and Android and the system's locale language. Also, couple of months ago, Google introduced the real time tracking, which allows the webmaster to monitor the visitors live, check their referring site and monitor the links they click and many more.

\section{1) Advantages}

Google Analytics, which is another great tool created by Google and provides an amazing insight on how the users are interacting with the website. Google analytics $[8,10]$ is mostly used by internet marketers to analyze the incoming traffic and measure its behavior. One of the biggest advantages that Google analytics offer is the ability to create and set goals that measure how well the website is fulfilling its objective. For example an ecommerce website has the goal of selling items, a goal can be created to trigger when a user purchase an item, this helps measuring the number of visitors against the number of buyers.

In addition Google Analytics provide ecommerce tracking that can track what users have bought, the quantity, revenue generated from each transaction, revenue of each product, total revenue, shipping, taxes, number of days and number of visits that a user have made before making their purchase.

Moreover, Google Analytics tracks the referring websites of every user, that is from which website a user came (Facebook, Twitter, Google, Email), the duration spent browsing the website, the pages visited, the bounce rate of users, the Operating System used, the Internet Service Provider used, the location (Country/City) and many more.

Furthermore, and to analyze the results more efficiently, Google Analytics results can be filtered by campaigns and destination source. For example, assuming we want to promote a product on Facebook, by creating Facebook advertisements and posting on a Facebook page, the destination source appearing on Google Analytics would be "Facebook" and the first campaign would be "fb-ads" and second campaign "fb-page". This helps analyze which campaign is doing better and which is driving more traffic and buyers.

Most of the Google Analytics Features can be used for free and can be used on any created website.

\section{2) Disadvantages}

The disadvantages of Google Analytics are the fact that sometimes they are inaccurate. This is because Google Analytics requires a webmaster to install a Javascript code in the website, usually inside the body tag (<body $\rangle$ ) in order to gather information about the visitors. The problem arises when a user or a visitor is using a browser that does not support Javascript, has Javascript disabled or has specific Javascript blocked. This mostly occurs from users with mobile devices, because not all browsers in mobile phones can run Javascript code. Also, many users have advertisements blocking add-ons installed on their browsers, usually those add-ons block third party Javascript codes, which is Javascript codes, that are included in the website's source code but are not part of the website's domain name.

All this will lead to inaccuracy in the data collected such as the inaccuracy in the number of visitors, the ecommerce transactions tracking, the goals set and the location and devices used. Google Analytics can provide a lot of information but should only be used when accuracy is not critical.

\section{Google PageSpeed Tool}

The speed of the website can have a huge influence on the search engine ranking. Reducing page load times can reduce bounce rates and increase conversion rates. Google provides a free tool to measure the page loading time. After a user enters the website's domain name in the text field and clicks analyze, Google will fetch the web page and provides a suggestion summary pointing out the high priority, medium priority and low priority (figure 6).

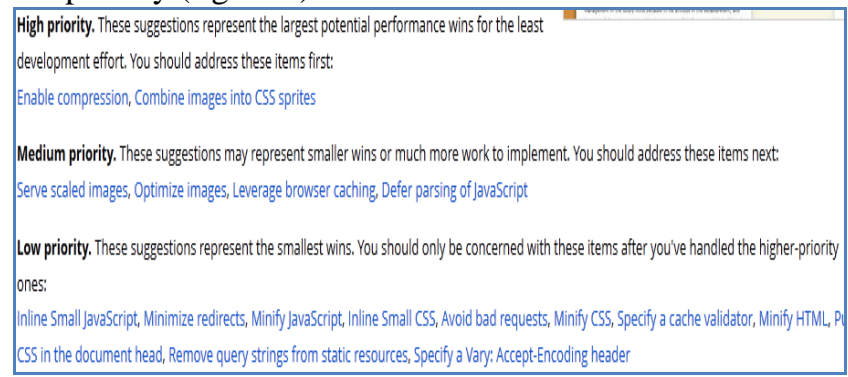

Fig. 6. Google PageSpeed - Suggestion Summary [11]

\section{1) Difference Between Priorities}

The high priority suggestions represent the largest potential performance wins for the least development effort, an example of high priority suggestions can be "Enabling Compression" when many CSS files and JavaScript files exist, or combining images into CSS sprites when many images are used on the 
website in order to reduce the server's load and increase the page speed.

The medium priority suggestions represent smaller wins or much more work to implement, an example of medium priority suggestions can be optimizing images in case an image has a very high resolution or is in bmp format which can slower the loading time and frustrate the user.

The low priority suggestions represent the smallest wins in increase the page speed, and those should only be concerned after the high priority and medium priority are handled. Most of the low priority suggestions are usually about minifying the JavaScript files, CSS files and HTML files. Minifying is the process of removing all the unnecessary characters from the source code. Such characters usually consist of space characters, new line characters, and comments. Usually those characters are used to make the code easier to read but they does not affect the execution of the code.

In addition, [11, 12] Google PageSpeed tools provide some experimental rules. These suggestions do not affect the overall PageSpeed score but can be useful to experiment new areas that might improve the overall speed of the website.

Google PageSpeed tools are very effective and can be helpful, because fast and optimized pages lead to higher visitor engagement, retention, and conversions.

2) Advantages

Google PageSpeed Tool measures the speed of a given website and provides helpful tips and tricks on how to decreases the page load time and hasten the request. The PageSpeed Tools provide a suggestion summary showing the highest priority issues, medium priority issues and low priority issues.

In addition, every issue that is slowing down the website is explained in details and a solution is given on what is the best practice to solve such issue.

Also, Google PageSpeed tools provide experimental suggestions. These suggestions do not usually affect the overall speed of the page but they can be useful to experiment new tactics that might improve the overall speed of the website.

If used correctly, Google PageSpeed can make a website faster and more optimized which leads to a higher visitor's engagement.

\section{3) Disadvantages}

Google PageSpeed Tool have some disadvantages as well. The primary disadvantage is it does not take into consideration the video and audio content. Many websites have videos and audio added to their website and those medias starts playing while the page is loading, which might result in a page taking much more loading time than the PageSpeed Tool has showed.

Another disadvantage is the bandwidth on Google servers compared to the bandwidth of the user. If a website contains a lot of high resolution images, Google might be able to load those pages way faster than an ordinary user can since servers in general have a much faster internet connection.

\section{D. Хепи Link Sleuth}

Xenu Link Sleuth is a great free tool that crawls any website in a similar way to search engines and quickly finds broken links and other problems. Xenu Link Sleuth follows all the links on the website and eventually check all the pages for broken links. Broken links can decrease the search engine rankings since there will be a lot of "page not found" errors.

In addition, it provides details and overview of the website's pages and provides suggestions on how to fix and improve them. Xenu Link Sleuth is very useful specially on new websites because it can help in discovering the simplest mistakes to the most complicated mistakes.

Xenu Link Sleuth $[13,164]$ can be downloaded for free and can be installed on any machine running a Windows operating system. The verification of the link is done by searching for the <a> HTML tags, images tags, and frames. It continuously updates the list of URLs until no new links are found. The below image displays Xenu Link Sleuth checking for broken links and displaying the broken links in red (figure 7).

\begin{tabular}{|c|c|c|c|c|c|}
\hline \multicolumn{5}{|l|}{ (G) Yenu- [Xenul] } & $\begin{array}{lll}-\square & \mathrm{x} \\
\end{array}$ \\
\hline 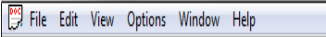 & & & & & $-5 x$ \\
\hline \multicolumn{6}{|l|}{ 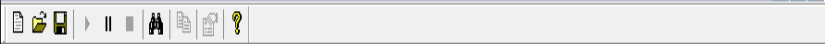 } \\
\hline Address & Status & Type & Size Title & Date & Level Out links = \\
\hline hitp:///wu.google.com/dud/cos/gsearch.css & ok & tedt/css & & $14.03,201302: 42: 38$ & 1 \\
\hline http://www:blogger, com/dyn-cs//uthorization, cssitargetbl|... & ok & $\operatorname{tet} / / s s$ & & $190032: 2013$ 00:00:26 & 1 \\
\hline hittp://themes.googleusercontentcom/imageilid:10ACCYO... & ok & image/peg & 80639 & $27.02: 201000: 13: 19$ & 1 \\
\hline http://wwviblogblog,com/1kt/transparent//whiteP0.png & ok & imzge/png & 96 & $12032,21319: 48445$ & 1 \\
\hline 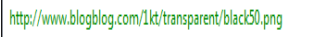 & ok & immge/png & 96 & $1203.201319 ; 4: 43: 45$ & 1 \\
\hline 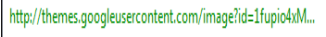 & ok & imsge/jpeg & 71706 & $260202201023: 18: 37$ & 1 \\
\hline 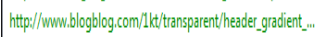 & ok & immge/png & 424 & $12032201319: 48: 45$ & 1 \\
\hline httpi//www.blogblog,com/likt/transporent/tabs_gradient_sha... & ok & $\mathrm{imagg/png}$ & 185 & 12032.2013 19:48:45 & 1 \\
\hline 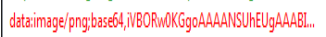 & notfound & & & & 1 \\
\hline http://wwwitech-and-dev.com/p/ariliceshtoml & ok & tert/html & 76725 Tech and $\mathrm{D}$.. & 11.03 .2013 10:55:49 & 178 \\
\hline
\end{tabular}

Fig. 7. Xenu - Checking Broken Links [14]

Xenu Link Sleuth [14] also supports websites with Secure Socket Layer (SSL) and can find all the broken links.

\section{1) Advantages}

Xenu Link Sleuth which is a totally free tool and is compatible with all Windows Operating Systems, even Windows 95. Xenu Link Sleuth works in a simple yet very effective way, it searches a page for all the HTML anchors <a> and updates a list of URLs, it works like Google by following all the links of a website till all the links have been processed.

Xenu Link Sleuth is a very effective and quick way in finding broken links in any website. Another great strong feature is the ability to support websites using SSL (https).

\section{2) Disadvantages}

Xenu Link Sleuth works by following links in a webpage. The first disadvantage is that it can only run on Windows Operating Systems, so a webmaster using Linux or Mac can never use this tool unless they have windows installed.

Because Xenu Link Sleuth is installed and running locally on the webmaster's computer, it might slow the internet connection or consume a lot of internet traffic specially for big websites since it's crawling links and checking for errors.

\section{E. Google AdWords Keyword Tool}

Probably the most important and well known keyword tool 
for websites, Google AdWords keyword tool allows one to find related keywords to the ones they enter and see how many searches all those keywords are receiving per month [15, 16, 17]. The results returned by Google AdWords Keyword tool contains information such as the competition on the keyword, the global monthly search of this keyword and the local monthly search.

Figure 8 shows a screenshot [18] of Google AdWords Keyword tool with the word "tech" entered in the search box.

\begin{tabular}{|c|c|c|c|c|c|c|}
\hline & Keyword & Competition & \multicolumn{4}{|c|}{$\begin{array}{c}\text { Global Monthly Searches Local Monthly Searches } \\
\text { ? }\end{array}$} \\
\hline$\square$ & tech 7 & Low & \multicolumn{2}{|c|}{$55,600,000$} & \multicolumn{2}{|c|}{$20,400,000$} \\
\hline \multirow[t]{2}{*}{ 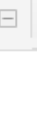 } & $\checkmark$ Save all & \multicolumn{2}{|c|}{ Keyword ideas (100) } & $1-50$ of 100 & $<$ & $>$ \\
\hline & \multicolumn{2}{|l|}{ Keyword } & Competition & $\begin{array}{c}\text { Global Monthly } \\
\text { Searches ? }\end{array}$ & \multicolumn{2}{|c|}{$\begin{array}{c}\text { Local Monthly } \\
\text { Searches? }\end{array}$} \\
\hline$\square$ & \multicolumn{2}{|c|}{ tech news } & Low & 201,000 & \multicolumn{2}{|c|}{74,000} \\
\hline$\square$ & \multicolumn{2}{|c|}{ tech skills $>$} & Low & 27,100 & \multicolumn{2}{|c|}{9,900} \\
\hline$\square$ & \multicolumn{2}{|c|}{ new tech gadgets } & Low & 2,400 & \multicolumn{2}{|c|}{1,600} \\
\hline
\end{tabular}

Fig. 8. Google AdWords Keyword Tool - Results [18]

According to the above picture, the global month searches on the word "tech" is around 55 million, and the local monthly search is around 20 million. Global Monthly Searches are the searches that were done by everyone in the world, while Local Monthly Searches are the searches that were done to the previously selected location that was chosen before clicking the search button. In this example, the United States were chosen as the location and thus the local monthly searches are the searches that were done on the keyword "tech" from the United States alone.

\section{1) The Importance of Competition Level}

The competition column is another very important column that can provide a lot of information. The competition can have three levels, High, Medium and Low. High competition is usually keywords that are receiving a massive amount of searches with a massive amount of websites targeting them [19]. Medium competition is the keywords that are receiving a high amount of searches with a medium amount of websites targeting them. Low competition are the keywords that are receiving a relatively high amount of searches, yet there are fewer websites out there targeting them.

Usually, new websites target keywords with low to medium competition level for two reasons, first reason being that those keywords do not have a lot of competition compared to the high level competition keywords, and second reason being that the high competition keyword levels are usually reserved for old sites that are well established and well optimized and competing with those websites might be very hard and may take ages for the results to appear and for the search engine rank to increase. In addition, the keyword search results can be easily exported to a CSV file for manipulation and studying.

The point of using the Keyword Tool is to determine which keywords are best used for a website and which keywords might rank higher with less competitiveness. After those keywords are found, they will be implemented on the website or blog by creating new content in the form of articles which mention these specific keywords in the page title, heading tags, body content, bold, italics, etc. The more they are mentioned, the better. It's believed that Google loves fresh, relevant content to the extent that they'll actually reward the website with a higher rank.

\section{2) Advantages}

Google AdWords Keyword Tool is the most important keyword tool for Google search engine. It allows one to analyze a specific keyword and find related keywords and see how many searches those keywords are receiving per month.

In addition, Google AdWords Keyword Tool will show information about the competition on the keyword and the amount of websites targeting this keyword worldwide or in a specific country [18]. This helps newly established websites by providing them enough information on which keywords they should target and which they should stay away from, this is because usually new websites can not compete as quickly with old well established ones. It also helps old websites to see how good their keywords are performing against their competitors.

Another advantage that Google AdWords have is the ability to export all the search results of keywords to a CSV file for later analyzing and studying.

\section{3) Disadvantages}

Google AdWords Keyword Tool have some similarity in the disadvantages as the Webmaster Tools. It's only useful to search and lookup keywords that works on Google search engine, which results in many other search engines being left out and potentially a large amount of visitors lost. For example a webmaster might find that a specific word has a low competition on Google search engine and decides to target it to gain more visitors, but that same word might have a high competition level on different search engines which might result in the website gaining more traffic from Google but losing some from other search engines.

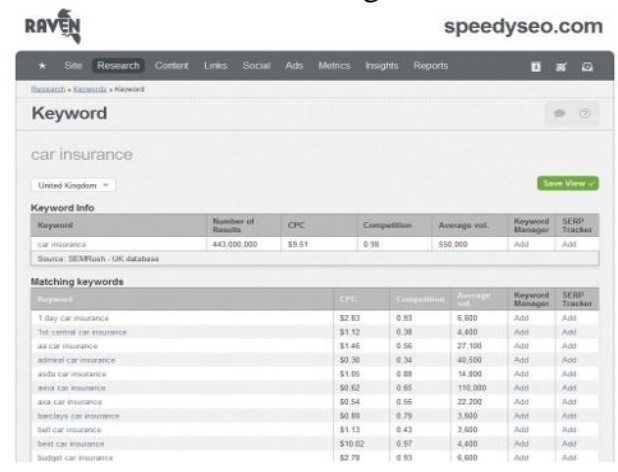

Fig. 9. Raven Tools Interface [20]

\section{F. Raven Tools}

Raven tools (figure 9) takes all the data that can be used in an online marketing campaign and brings it all together in one place.

Raven Tools is an all in one SEO tool. The list of included tools are so long that it makes it hard to get familiar to it quickly. 
Raven tools includes research tools to search for keywords and the competition of those keywords and the monthly search, in addition it includes contact managers, keyword managers, use data from the Google AdWords campaigns such as reports and insights, grab Google Analytics data from the account and even access the system via their own free iOS app.

It can also be used with a group of users to assign every user different permissions. A user can be given access to only one part of the tool such as Google Analytics data. Those users can also be assigned different tasks with little effort, allowing the webmaster to fully manage the SEO campaign among the team.

Raven tools also includes tools for social media, such as Facebook and Twitter, tools for Content Management Systems (CMS) such as Wordpress and Joomla, and support for third party applications such the famous Mailchimp which is used for sending email campaigns and delivering reports.

\section{1) Advantages}

Raven Tools is basically an all in one SEO tool that contains many different tools to help a webmaster analyze and promote their website from one single application.

Raven tools provides tools to search for keywords and displays the competition of those keywords and the monthly search done.

Raven tools can import reports and insights from Google AdWords and Google Analytics campaigns. It can also be used with a group of users to assign every user a different permission.

In addition, Raven tools allows to control social media, such as Facebook and Twitter, and to control Content Management Systems (CMS) such as Wordpress and Joomla, and support for third party applications.

\section{2) Disadvantages}

Raven Tools has many disadvantages. Because it's an all in one SEO tool that has many options, features and tools, more than a webmaster actually needs, it might be very difficult to learn and adapt to all the tools, and hard to keep track and maintain them.

Raven tools does not delve as deeply into the provided tools and only offers some of the basic features from every tool. This can be useful for people that are new to the SEO field to get a glance on how everything works but it can be useless for professionals since it lacks many options and features that the original tools offer.

Another disadvantage is, some services supported by Raven Tools gets updated frequently which means that Raven tools has to be updated as well. If the updates for Raven Tools are not done as quickly as possible, the webmaster will be forced to either wait or use the original tool which also means that the competitors might eventually get one step ahead because they are adopting new technologies faster.

\section{G. Alexa}

Alexa is a website ranking system created by amazon.com, it's main goal is to determine how popular a website is compared to other websites.
The Alexa traffic rank $[20,21,22,23]$ is taken from data collected from millions of users over a period of the last three months. The collected information is based on the number of users who visited a specific website and the number of pages that were viewed by that same user. The traffic rank is updated almost everyday depending on the page views and website visits, the lower the Alexa number rank is the higher the amount of traffic a website is receiving, for example a website ranked in position 1 would indicate that this website have the highest rank that any website can reach.

The traffic rank is based on the collected historical data from millions of installed Alexa Toolbars on user's machines. In addition, the data is also obtained from other traffic sources. The traffic rank is a combination of page views and number of visitors. The initial step consists of a complex algorithm that computes the number of visitors and the number of page views for all the websites on the World Wide Web on a daily basis [23]. The Alexa traffic rank is derived from the results of this calculation averaged over time, so that the site rank is eventually reflecting both the number of visitors and the number of users who visited this website.

Alexa provides many free tools to potentially increase the website rank. The most important tools are the Alexa Toolbar and the Alexa Widget.

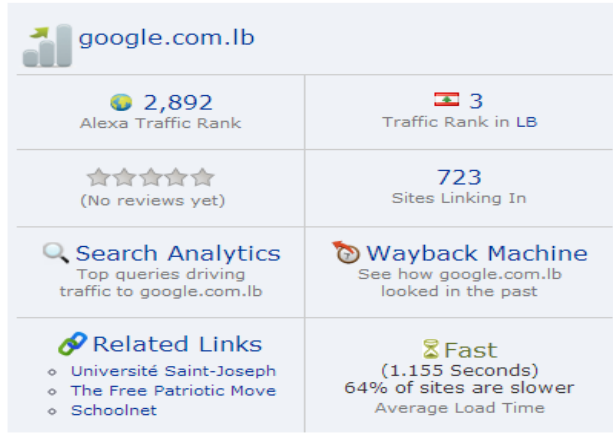

Fig. 10. Alexa Toolbar [23]

\section{1) Alexa Toolbar}

Alexa toolbar (figure 10) [25] is a free downloadable plugin for browsers, it allows one to measure their own website's traffic and rankings and to check out other websites ranking. The toolbar also provides the user with a way back machine which shows how the website looked like months or years ago. In addition it shows the average load time of the webpage and compares our website load time and performance with other websites. Downloading and installing the Alexa toolbar is very straightforward, it's as easy as installing any plugin for the browser and does not require any configuration.

\section{2) Alexa Widget}

The Alexa Widget [23] is added to the website's source code, it shows the website's worldwide rank and if chosen the number of links pointing to this website. When a visitor access a website with the Alexa widget installed, Alexa will collect more precised data which can increase the website's rank and provides more historical information. All of this information is very valuable because it helps the webmaster to conclude how the website and other sites are performing and eventually 
improve this website to attract more users.

\section{3) Advantages}

Alexa, created by amazon.com to determine the popularity of a website compared to others. Alexa collects data from millions of users from different sources, and the ranking is based number of visitors and number of pages views per visitor. Because Alexa is very famous, having a better rank can indicate which website is better among a group of competitors and which website is getting more visitors and a better reputation.

In addition to ranking, Alexa [23] provides a very important tool as a toolbar for browsers. The toolbar has many advantages such as providing a way back machine that shows how the website looked months or years ago. The toolbar also shows the average load time of a webpage and compares a website with another. Furthermore, Alexa offers a search analytics that shows the search queries that were used by users to find the selected website, the location of those users, manually submitted reviews, related links and similar websites and most importantly the sites linking to the selected website. Alexa toolbar is very easy to install, configure and use.

\section{4) Disadvantages}

Alexa, which is used to determine the popularity of a website compared to others has also many disadvantages. This is because not everybody has the Alexa toolbar installed, which is used to collect data about the visited websites and the time spent on that website. Although, Alexa relies on different sources of information other than the toolbar, but the toolbar can greatly influence the information collected which means that websites visited by users that have the toolbar installed will eventually rank better than the ones without the toolbar installed, so the popularity of a website might not be very accurate.

Many people believe that the Alexa ranking are not important and can be greatly influenced (or "gamed" as some prefer to call it), even though there is no actual proof that this is the case. The overall traffic provided by Alexa is based on the top domain only. In other words, sub-domains and subpages are not ranked separately, which can limit the amount of information and analysis for a website with many sub-domains and subpages.

\section{CONCLUSIONS}

In this paper, we introduced some of the available solutions to the Search Engine Optimization problems and their advantages and disadvantages. Different types of solutions exist and are developed, but few are practical enough to enhance the search engine optimization of a website or to provide helpful tips on how to improve the website.

\section{ACKNOWLEDGMENT}

This work has been done as a part of the project " Search Engine Optimization (SEO) For Websites" by Lebanese
University.

\section{REFERENCES}

[1] Ho Li-Hsing, Huang Jui-Chen, Lu Meng-Huang and Ho Hui-Yi, "The application of search engine optimization for internet marketing: An example of the motel websites" in Computer and Automation Engineering (ICCAE), 2010, pp. 380-383.

[2] Wu Di, Luan Tian, Bai Yan, Wei Liyuan and Li Yanhui, "Study on SEO monitoring system based on keywords \& links" in Computer Science and Information Technology (ICCSIT), 2010, pp. 450-453.

[3] Austin Richard, "Useful SEO Techniques to Achieve High Results", 2013

[4] Ao-Jan Su, Hu, Y.C., Kuzmanovic, A. and Cheng-Kok Koh, "How to Improve Your Google Ranking: Myths and Reality" in Web Intelligence and Intelligent Agent Technology (WI-IAT), 2010, pp. 50-57.

[5] Fuxue Wang, Yi Li and Yiwen Zhang, "An empirical study on the search engine optimization technique and its outcomes" in Artificial Intelligence, Management Science and Electronic Commerce (AIMSEC), 2011, pp. 2767-2770.

[6] Janet Driscoll Miller, "Using The Structured Data Report In Google Webmaster Tools", SearchInsider, 2012.

[7] Google, Google Webmaster Tools [computer software], 2013, http://www.google.com/webmasters/tools, accessed on 16/03/2013

[8] Google, "Analysis of Users' Behavior on Web 2.0 Social Network Sites: An Empirical Study" in Information Technology: New Generations (ITNG), 2010, pp. 454 - 459

[9] M. Chafkin, "Analyze this, says Google," Inc., vol. 28, Apr. 2006, pp. 30-30.

[10] Google, Google Analytics [computer software], 2013, http://www.google. com/analytics, accessed on 17/03/2013.

[11] Google, Google PageSpeed [computer software], 2013, https://developers.google.com/speed/pagespeed/insights, accessed on $19 / 03 / 2013$

[12] Mo Yunfeng, "A Study on Tactics for Corporate Website Development Aiming at Search Engine Optimization" in Education Technology and Computer Science (ETCS), 2010, pp. 673-675.

[13] Ian Harac, "Xenu's Link Sleuth Finds Broken Web Site Links and Images", PC World Magazine, 2009.

[14] Tilman Hausherr, Xenu Link Sleuth [computer software], 2013, http://home.snafu.de/tilman/xenulink.html, accessed on 21/03/2013.

[15] Di Di, Luan Tian, Bai Yan and Wei Liyuan "Study on SEO monitoring system based on keywords \& links" in Computer Science and Information Technology (ICCSIT), 3rd IEEE International Conference, 2010, pp. 450-453.

[16] Huanwei Wu, "Search Engine Optimization of E-Commerce Websites" in Management and Service Science (MASS), International Conference, 2011, pp. 1-3.

[17] Mason Zachary, "WordRank: A Method for Finding Search-Ad Keywords for Internet Merchants" in Internet and Web Applications and Services, 2007, pp. 12.

[18] Google, Google Adwords Keyword Tool [computer software], 2013, http://adwords.google.com/, accessed on 24/03/2013

[19] Mehta A., Saberi A., Vazirani U. and Vazirani V., "AdWords and generalized on-line matching " in Foundations of Computer Science, 2005, pp. 264-273.

[20] Raven, Raven Tools [computer software], 2013, http://raventools.com, accessed on 24/03/2013.

[21] Lin Li, "Social network sites comparison between the united states and China: Case study on facebook and renren network" in Business Management and Electronic Information (BMEI), 2011, pp. 825-827.

[22] Yihong Zhan and Yanyan Yan "Construction and Optimization of Recruitment Websites in China" in Business Intelligence and Financial Engineering (BIFE), 2013, pp. 143-146.

[23] Amazon, Alexa [computer software], 2013, http://www.alexa. .com, accessed on 25/03/2013. 